\title{
Linee guida per l'assistenza aziendale nella realizzazione dei solchi acquai efficaci nel contenimento dell'erosione (Standard di Condizionalità 1.1a) nell'ambito della Misura M02, Sottomisura 2.3 (Sostegno alla formazione dei consulenti) dei PSR
}

\author{
Paolo Bazzoffi \\ CREA-ABP, Consiglio per la Ricerca in Agricoltura e l'Analisi dell'Economia Agraria, Centro di Ricerca per l'Agrobiologia e la \\ Pedologia, Firenze, Italia
}

Autore corrispondente: Paolo Bazzoffi

E-mail: paolo.bazzoffi@@crea.gov.it

Parole chiave: Indicatori; condizionalità; standard; erosione; solchi acquai.

Lavoro svolto nell'ambito del Progetto MO.NA.CO. (Rete di monitoraggio nazionale dell'efficacia ambientale della condizionalità e del differenziale di competitività da essa indotto a carico delle imprese agricole) finanziato dal Ministero delle Politiche Agricole, Alimentari e Forestali (MiPAAF) nell'ambito del Programma Rete Rurale Nazionale nel contesto dell'Azione 1.2.2 "Laboratori interregionali per lo sviluppo" del Programma Operativo denominato "Rete Rurale Nazionale 2007 - 2013 Coord. Paolo Bazzoffi".
(C) Copyright P. Bazzoffi, 2015

Licenziatario PAGEPress, Italy

Italian Journal of Agronomy 2015; 10(s1):719

doi:10.4081/ija.2015.719

Questo articolo è distribuito secondo i termini della licenza Noncommercial Creative Commons Attribution (by-nc 3.0) che permette qualsiasi uso non commerciale, la distribuzione e la riproduzione con qualsiasi mezzo, a condizione che l'autore (autori) originale (i) e la fonte siano accreditati.

\section{Premessa}

La misura M02 implementata dai PSR 2014-2020 ha l'obiettivo di aiutare gli imprenditori agricoli e forestali ad avvalersi dei servizi di consulenza per migliorare le prestazioni economico ambientali delle loro imprese. La misura incoraggia inoltre la partecipazione dei destinatari dei servizi ad attività di consulenza finalizzate ad accrescerne le competenze professionali in materia di tutela, ripristino e valorizzazione degli ecosistemi connessi all'agricoltura e alla selvicoltura e incentivare l'uso sostenibile delle risorse.

La M02 si compone di 2 sottomisure:

a) Sottomisura 2.1 Sostegno allo scopo di aiutare gli aventi diritto ad avvalersi di servizi di consulenza

b) Sottomisura 2.3 Sostegno alla formazione dei consulenti

Dalle finalità della M02 deriva la necessità che i servizi di consulenza forniscano all'agricoltore le informazioni necessarie al raggiungimento degli obbiettivi ambientali fissati dalla PAC.

Fra le Norme e gli Standard BCAA di condizionalità del decreto MiPAAF del 2009 e seguenti ( fino al recente decreto n. 180 del 23 gennaio 2015) per il mantenimento dei terreni in buone condizioni agronomiche e ambientali che mirano all'Obiettivo 1 - Erosione Del Suolo: Proteggere il suolo mediante misure idonee, è compresa la Norma 1: "Misure per la protezione del suolo", che si articola in diversi standard fra cui lo standard 1.1: Gestione minima delle terre che rispetti le condizioni locali specifiche e lo standard 1.2: Copertura minima del suolo.

Lo standard $1.1_{\text {(impegno a) }}$ (solchi acquai temporanei) e lo standard 1.2 (impegno h subv.) (adottare tecniche per la protezione del suolo in alternativa alla copertura vegetale autunno vernina) sono impegni resi obbligatori dalla Condizionalità al fine della riduzione dell'erosione del suolo nei terreni agricoli. In modo particolare, la realizzazione dei solchi acquai temporanei può suscitare notevoli difficoltà decisionali nell'agricoltore al quale è richiesto dalla Condizionalità di favorire la protezione del suolo dall'erosione ed allo stesso tempo che i solchi mantengano la loro funzione di emungimento delle acque di scorrimento superficiale, con- vogliandole nei fossi collettori; ovvero mantengano la loro efficienza idraulica durante tutto il periodo nel quale può prodursi l'erosione del suolo. L' attuale standard $1.1_{\text {(impegnoa) }}$ (d'ora in avanti abbreviato in standard 1.1 a) non reca indicazioni particolari riguardo alla modalità di esecuzione dei solchi acquai, lasciando all'agricoltore l'arduo, se non impossibile, compito di determinare la spaziatura e la profondità ottimale dei solchi. D'altra parte occorre dire che fino al presente la letteratura scientifica e i manuali tecnici hanno fornito indicazioni assai vaghe in tal senso. Ciò si è ripercosso sulla normativa di Condizionalità Nazionale e Regionale, che non ha fornito all'agricoltore le indicazioni idonee alla realizzazione di solchi acquai temporanei efficaci a regimare i picchi di deflusso e l'erosione del suolo. I solchi acquai, se eseguiti a regola d'arte (Figura 1), ovvero con profondità adeguata a recepire il deflusso e con inclinazione rispetto alla linea di massima risultano efficaci nella regimazione dei deflussi e di contenimento dell'erosione. Ciò è stato dimostrato dal monitoraggio sia nell'ambito del progetto MO.NA.CO. (Bazzoff et al., 2015) sia nelle precedenti sperimentazioni condotte in Italia in prove di campo parcellari. I solchi assolvono anche ad un'altra importantissima funzione, ovvero consentono al materiale solido in sospensione, presente nelle acque di ruscellamento e incanalato nei rigagnoli, di depositarsi nel solco stesso, mantenendo sul versante il materiale asportato e diminuendo, di conseguenza, l'apporto solido al reticolo idrografico. Nel presente lavoro si riportano anche alcuni risultati relativi all'effetto di diversi tipi di lavorazione del suolo sull'erosione, in relazione agli ulteriori impegni agronomici imposti dalla condizionalità ad integrazione dell'obbligo di realizzazione dei solchi acquai temporanei.

\section{Errori frequenti nell'esecuzione dei solchi acquai}

Molto frequentemente si osserva che i solchi acquai vengono eseguiti in modo da risultare inefficaci o, peggio ancora, da aggravare l'ero- 
sione del suolo invece che diminuirla.

Alcuni dei più comuni errori sono i seguenti:

1) Solchi realizzati negli impluvi, anzichè con andamento trasversale rispetto alla massima pendenza e pertanto inefficaci (Figura 2).

2) Solchi realizzati con andamento lungo la massima pendenza anziché in senso trasversale. Non potendo intercettare il deflusso risultano inefficaci (Figura 3).

3) Solchi non sufficientemente profondi, realizzati con l'aratro a versoio anziché con lo scavafossi. I solchi possono regimare picchi di deflusso di modesta intensità mentre, per eventi intensi, potrebbero collassare determinando effetti rovinosi a valle (Figura 4).

4) Solchi che in un punto del loro percorso procedono in contropendenza creando una concentrazione del deflusso e l'interrimento del solco medesimo per deposizione del materiale terroso in sospensione. Ne consegue la rottura dell'argine del solco acquaio con incremento dell'erosione, talvolta rovinosa (Figura 5).

5) Sottodimensionamento o eccessiva distanza fra solchi, per cui i solchi non sono in grado di recepire tutto il deflusso, con effetto di concentrazione dell'erosione "domino" (Figura 6).

L'acquisizione del know-how necessario all'esecuzione di solchi acquai efficaci, in numero, localizzazione e dimensioni può avvenire soltanto attraverso un servizio di consulenza aziendale in grado di fornire indicazioni idonee e non approssimative.

Le presenti linee guida sono state formulate per colmare queste lacune di conoscenze e vengono fornite ad uso principale dei Servizi di Consulenza al fine di guidare gli agricoltori nella corretta applicazione dello standard di condizionalità $1.1_{\mathrm{a}}$.

\section{Fondamenti teorici per la progettazione}

\section{Determinazione dei picchi di deflusso che devono essere regimati dai solchi acquai}

Per valutare l'efficacia dei solchi acquai a regimare i deflussi interessa conoscere i volumi di acqua che la scolina dovrà smaltire in momenti di picco (Landi et al., 1982).

I cambiamenti climatici in atto, caratterizzati da un aumento di frequenza degli eventi estremi (Scoccimarro, 2013; Bazzoffi e Pellegrini, 1992; Buffoni et al., 2003), consigliano di dimensionare l'affossatura primaria considerando i picchi di deflussi piuttosto che i valori medi annuali. A questo proposito occorre dire che in Italia le osservazioni sperimentali sono scarse e le poche ricerche a scala parcellare o di versante, sia quelle concluse in passato sia quelle in corso, sono state finalizzate alla messa a punto e allo sviluppo della modellistica dell'erosione su aree ristrette del territorio italiano, I dati pubblicati nell'ambito di queste ricerche mostrano i valori dei volumi di deflusso totali generati dagli eventi piovosi e non riguardano i picchi di deflusso occorsi durante gli eventi, Poche sono le ricerche finalizzate alla verifica diretta della funzionalità dei fossi acquai.

In genere in Italia l'affossatura viene dimensionata utilizzando alcune formule per il calcolo del picco di deflusso, fra le quali la più nota è il "metodo razionale":

$$
\mathrm{Q}=0,0028 \mathrm{C} \cdot \mathrm{I} \cdot \mathrm{A}
$$

Dove $Q$ è il picco di deflusso $\left(\mathrm{m}^{3} / \mathrm{sec}\right)$ dal bacino; $\mathrm{C}$ è il coefficiente di deflusso (adimensionale); I è l'intensità della pioggia ( $\mathrm{mm} / \mathrm{h}$ ) uguale al tempo di concentrazione del bacino; A è l'area del bacino (ha). Nell'utilizzo di questa formula si presuppone che l'intensità della pioggia sia costante ed uguale al tempo di concentrazione del bacino.

Il metodo razionale risulta sempre del tutto affidabile; invece risulta-

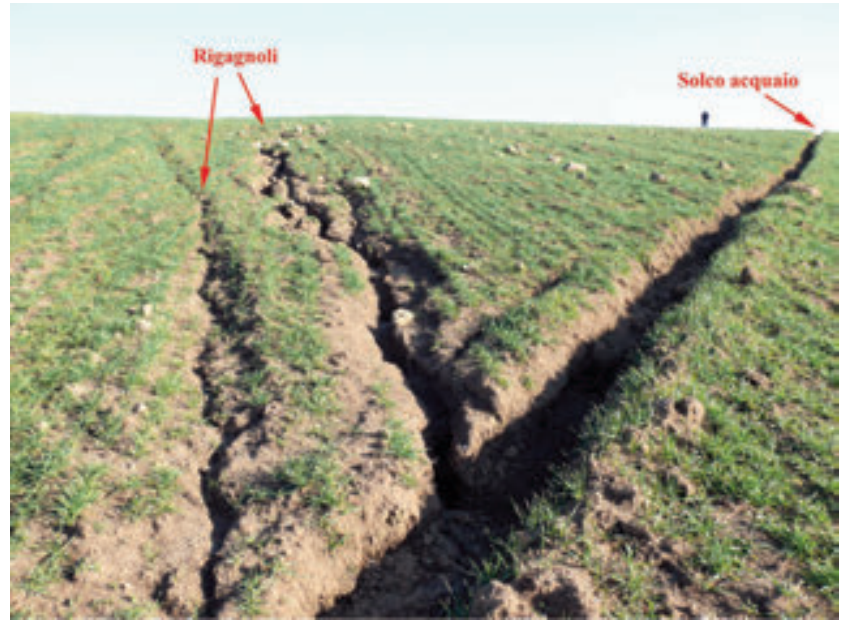

Figura 1. Solco acquaio eseguito a regola d'arte, con adeguata profondità ed inclinazione rispetto alla massima pendenza. Il deflusso dei rigagnoli viene totalmente intercettato dal solco.

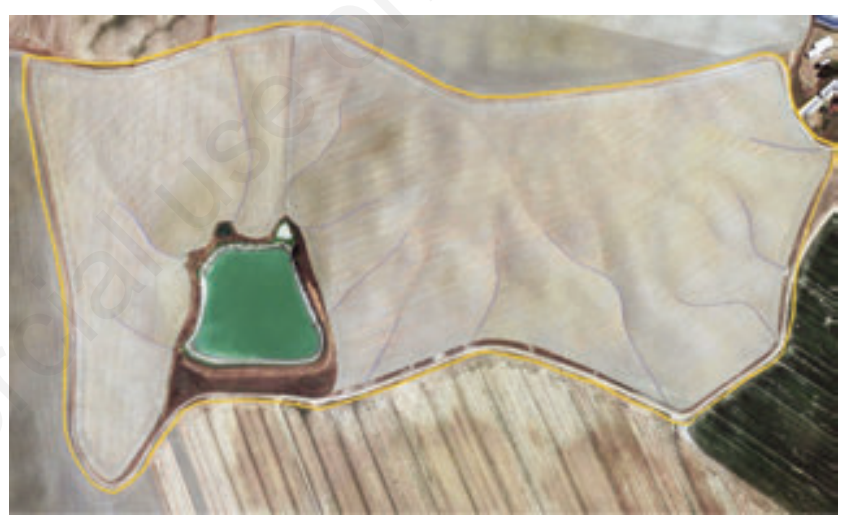

Figura 2. Immagine satellitare di solchi acquai inefficaci, perché realizzati negli impluvi naturali anzichè con andamento trasversale rispetto alla massima pendenza del versante.

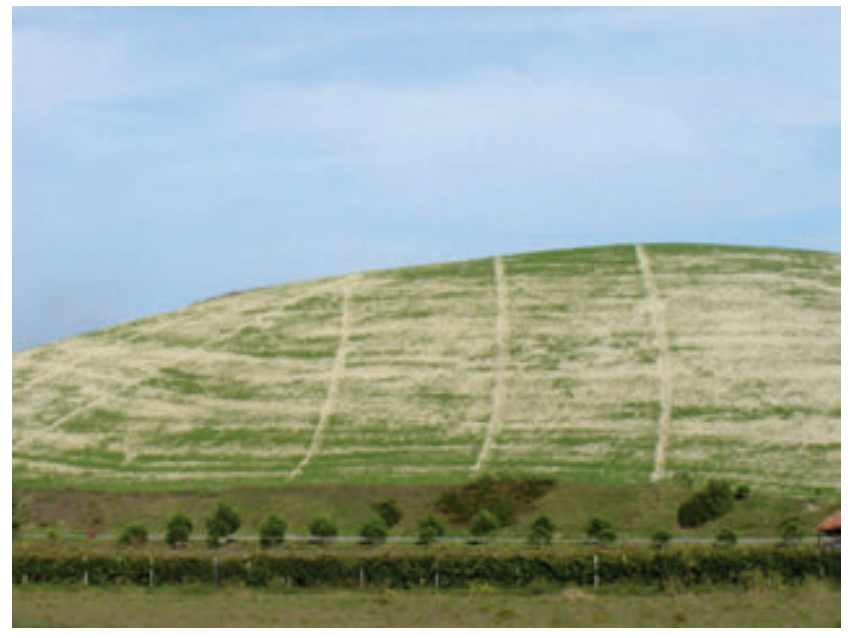

Figura 3. Solchi acquai inefficaci perché realizzati con andamento lungo la massima pendenza anziché in senso trasversale. 
no più interessanti i dati su afflussi e deflussi che sono stati reperiti facendo riferimento alla ricerche condotta in Italia da vari enti di ricerca a partire dagli anni ' 70 fino al presente.

A Figline Valdarno, nell'ambito del sub-progetto Dinamica dei versanti del progetto finalizzato Conservazione del suolo del CNR è stata condotta una sperimentazione che ha fornito dati sui picchi di deflusso (Landi et al. 1982), Essa ha riguardato un terreno limo sabbioso (58,7\% di sabbia) con pendenza media del 16\%, ove sono stati rilevati i dati di deflusso su quattro grandi parcelle isolate idraulicamente, larghe $20 \mathrm{~m}$, lunghe rispettivamente 80 e $50 \mathrm{~m}$ e lavorate a 50 e $30 \mathrm{~cm}$.

In quest'aerea, l'analisi sui picchi di deflusso per l'evento critico del 4 novembre 1980 (in seguito ad un massimo di precipitazione che ha raggiunto i $39 \mathrm{~mm}$ in un'ora) ha mostrato un valore massimo pari a $0,037 \mathrm{~m}^{3} \mathrm{~s}^{-1} \mathrm{ha}^{-1}$ sulle parcelle lavorate a $50 \mathrm{~cm}$, mentre dalle parcelle lavorate a $30 \mathrm{~cm}$ il picco di deflusso è stato di ben $0,071 \mathrm{~m}^{3} \mathrm{~s}^{-1} \mathrm{ha}^{-1}$.

A Guiglia (Modena) l'ex Istituto Agronomico (oggi CRA) ha condotto una serie di ricerche dal 1976 al 1983 su due piccoli bacini uniformi di 1,5 ha con 13\% di pendenza (suoli argillosi -Vertic Eutrochrept), Il massimo picco di deflusso è stato osservato in questa stazione sperimentale nell'evento del 22 giugno 1981, con un valore di $0,0333 \mathrm{~m}^{3} \mathrm{~s}^{-1} \mathrm{ha}^{-1}$ (Chisci e Boschi, 1988). Questi valori sono in linea con quelli osservati a Vicarello (Volterra) presso l'azienda sperimentale del CRA-ABP, nell'ambito del progetto Finalizzato Mipaaf "PANDA" ed in progetti di ricerca successivi, per un totale di 10 anni di registrazioni, In quest'area, su vertisuoli a tessitura franco-limoso argillosa di origine pliocenica, sono presenti, fin dagli anni ' 60 , 8 parcelle idraulicamente isolate ed attrezzate che raccolgono i deflussi da un intero versante, Le

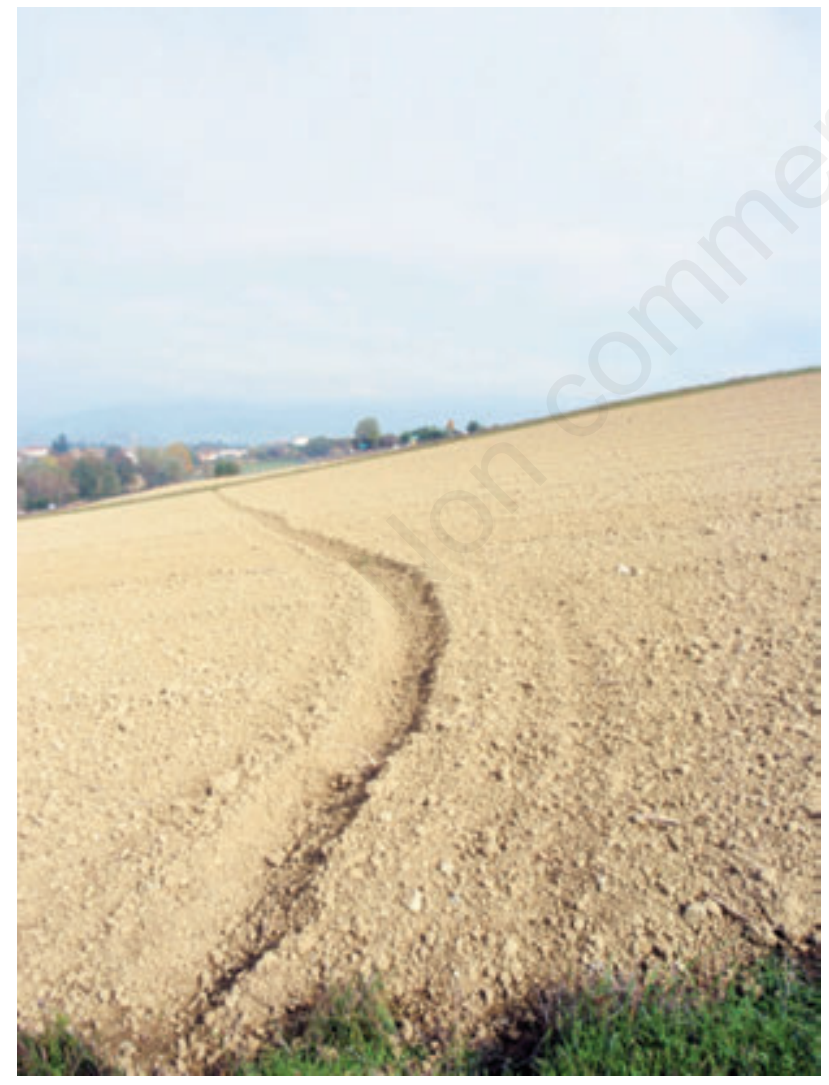

Figura 4. Solco acquaio di scarsa profondità realizzato con l'aratro a versoio anziché con lo scavafossi. Il solco appare efficace solo nel contenimento di picchi di deflusso di modesta intensità. Per eventi piovosi più intensi l'argine del solco potrebbe collassare determinando effetti rovinosi a valle.

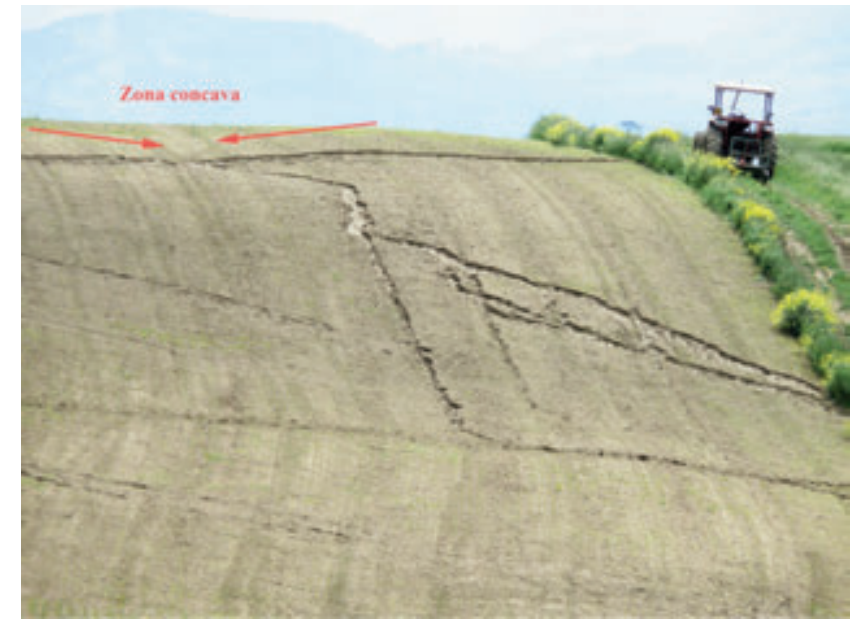

Figura 5. Rottura del solco acquaio con incremento dell'erosione a valle. In corrispondenza del punto di rottura il solco è stato realizzato in una zona leggermente concava (indicata dalle frecce).

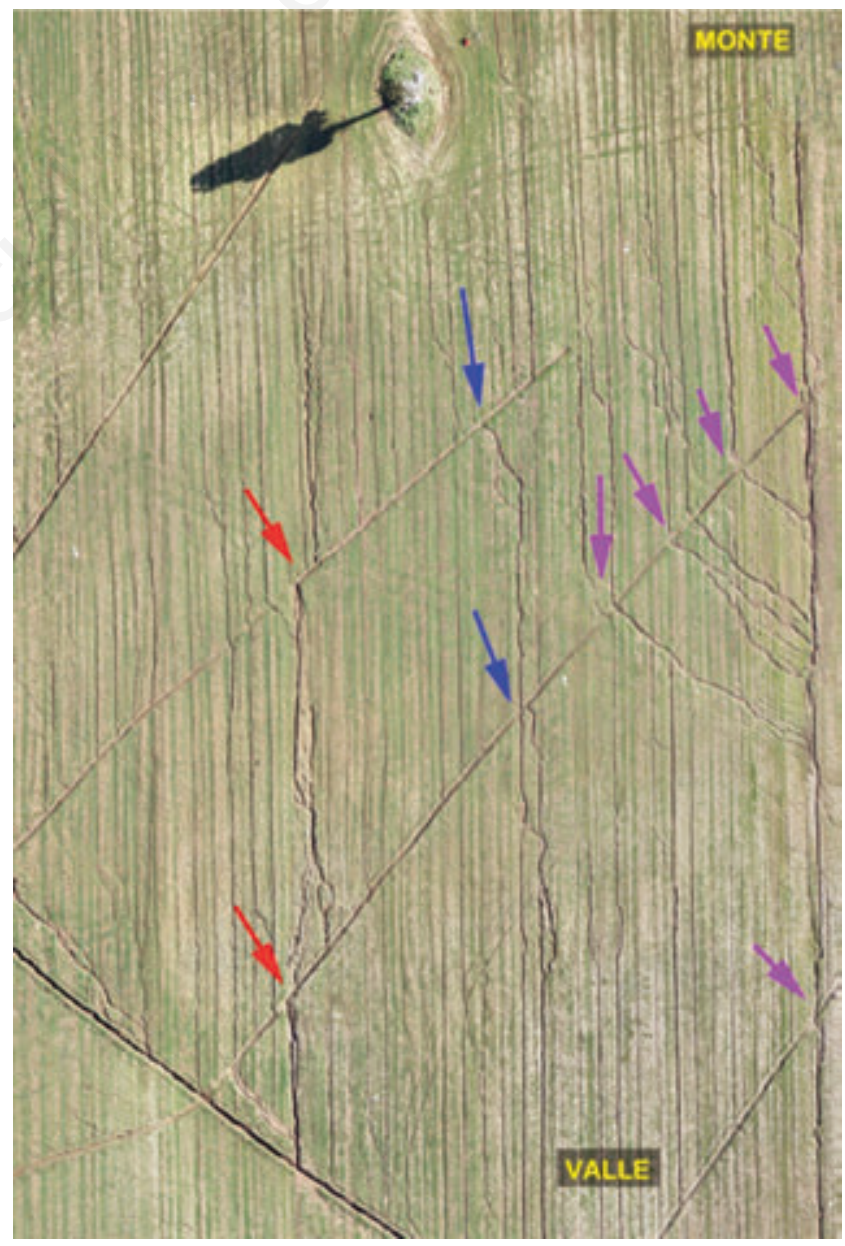

Figura 6. Sottodimensionamento o eccessiva distanza fra solchi, per cui i solchi non sono in grado di recepire tutto il deflusso, con effetto di concentrazione dell'erosione "domino". Le frecce di diversi colori identificano i diversi rigagnoli e indicano le zone dove il deflusso non è stato intercettato efficacemente dai solchi acquai. 
parcelle, lunghe $75 \mathrm{~m}$ sulla massima pendenza e larghe $15 \mathrm{~m}$, sono state attrezzate con unità idrologiche elettroniche di registrazione dei deflussi di estremo dettaglio.

Si richiama l'attenzione sul fatto che il valore medio di deflusso ed il picco massimo (Tabella 1) sono risultati rispettivamente più bassi di 3,13 e 2,7 volte rispetto ai valori stimati con il metodo razionale Il picco massimo è stato osservato per un evento piovoso eccezionale di 56,8 mm verificatosi in data 19/02/1996, con intensità massima in 2 minuti pari a $54 \mathrm{~mm} / \mathrm{h}$. Contemporaneamente alle misure parcellari, sempre a Vicarello, sono stati monitorizzati due sottobacini monocolturali di 4,66 ha e 5,62 ha, utilizzati rispettivamente a erba medica (prato di vecchio impianto) e a grano duro I massimi picchi di deflusso sono stai osservati nei due anni 1993-1994, con valori di 0,031 e di 0,037 $\mathrm{m}^{3} \mathrm{~s}^{-1} \mathrm{ha}^{-1}$, rispettivamente per il bacino a erba medica e per il bacino a grano duro. In una precedente indagine condotta dal CREA-ABP (ex ISSDS) fra il 1971 e il 1972 su due piccoli bacini del paesaggio calanchivo del Volterrano, rispettivamente di $1,06 \mathrm{~km}^{2}$ e di $0,91 \mathrm{~km}^{2}$, i picchi massimi osservati sono stati di 0,0191 e 0,0193 $\mathrm{m}^{3} \mathrm{~s}^{-1} \mathrm{ha}^{-1}$ (Bazzoffi et al., 1997).

In una ricerca condotta dal 1975 al 1979 (Zanchi, 1981) presso l'azienda del CREA-ABP a Fagna (Scarperia, Firenze), su un intero versante con pendenza media del 12,4\% con suolo argilloso, furono osservati i seguenti deflussi massimi: $0,0022 \mathrm{~m}^{3} \mathrm{~s}^{-1} \mathrm{ha}^{-1} ; 0,0057 \mathrm{~m}^{3} \mathrm{~s}^{-1} \mathrm{ha}^{-1} \mathrm{e}$ $0,0060 \mathrm{~m}^{3} \mathrm{~s}^{-1} \mathrm{ha}^{-1}$ su di tre parcelle rispettivamente con le seguenti tesi: Pascolo con carico normale, Sovrapascolato e Sfalciato.

In Sicilia, a Sparacia nei pressi di Palermo, gli studi condotti su un piccolo bacino di 3,67 ha con pendenza media del 19,5\% e suoli argillosi hanno evidenziato nel periodo 2004-2006 un picco massimo di deflusso di $0,0046 \mathrm{~m}^{3} \mathrm{~s}^{-1} \mathrm{ha}^{-1}$ (Di Stefano et al., 2008).

In Calabria, nel Crotonese, in un piccolo bacino calanchivo di 1,47 ha con $35 \%$ di pendenza media, sono stati monitorati i deflussi nei due periodi dal 1978 al 1994 e dal 2008 ad oggi Il valore più elevato di deflusso è stato osservato in data 26-09-2009 per un valore pari a $0,171 \mathrm{~m}^{3}$ $\mathrm{s}^{-1} \mathrm{ha}^{-1}$ (com. pers. di Paolo Porto dell'Università degli Studi "Mediterranea" di Reggio Calabria).

L'Accademia di Agricoltura a Vezzolano (T0) dal 1992 al 1998, su terreno limoso-argilloso, ha effettuato ricerche sui deflussi in un vigneto posto su un versante con pendenza di circa il $10 \%$. Nel vigneto sono state isolate idraulicamente due parcelle di circa $5500 \mathrm{~m}^{2}$. Nei sette anni di osservazioni sono stati analizzati 101 eventi con deflussi superiori a 3001 ha $^{-1}$. Il picco di deflusso massimo è stato osservato nell'evento critico del 25 settembre 1993, con un valore di $12,2 \mathrm{~mm} \mathrm{~h}^{-1}$, pari a $0,0339 \mathrm{~m}^{3} \mathrm{~s}^{-1} \mathrm{ha}^{-1}$ (Lisa e Parena, 1994).

Elaborando i dati raccolti si è ottenuta un'equazione per la stima del picco massimo di deflusso in relazione all'area del bacino e alla pendenza (\%), valida per il territorio italiano, come segue:

$$
p=e^{a+b \cdot \ln x+c y^{3}}
$$

dove $p=\mathrm{m}^{3} \mathrm{~s}^{-1} \mathrm{ha}^{-1} ; \mathrm{x}=$ area in ha; $\mathrm{y}=$ pendenza media $\%$.

Nella Tabella 2 si riporta il grafico dell'equazione e l'analisi statistica del modello con i valori dei parametri a, b, c dell'equazione 2 .

\section{Determinazione della dimensione e spaziatura dei solchi acquai}

Pochi sono i testi in cui si riportano osservazioni precise circa il dimensionamento opportuno della rete scolante per l'ambiente italiano. Tra questi citiamo Gasparini $(1959,1970)$ e Landi (1978) che per le fosse livellari indicano i seguenti parametri tecnici: profondità della fossa livellare di $5-10 \mathrm{~cm}$ superiore alla profondità di lavorazione; distanze tra le fosse di 60-100 m: pendenze di 1,5-2,5\%; sviluppo lineare del fosso non superiore ai $200 \mathrm{~m}$; presenza di una rete acquidocci e compluvi naturali in cui convogliare le acque raccolte dalle fosse livellari.

Per valutare l'efficienza dei fossi acquai è necessario confrontare la loro capacità massima di emungimento del deflusso $\left(\mathrm{m}^{3} \mathrm{sec}^{-1}\right)$ con i valori di picco di deflusso massimo, calcolati con la formula (2); considerando l'area della superficie scolante compresa fra due solchi acquai contigui, in funzione della lunghezza del solco e della pendenza del versante.

Per il calcolo della portata massima di emungimento da parte del solco si sono applicate le formule di Chézy e Bazin come segue:

Chézy: $Q=C \cdot A \sqrt{R \cdot I}$

Bazin: $\quad C=\frac{87 \cdot \sqrt{R}}{\gamma+\sqrt{R}}$
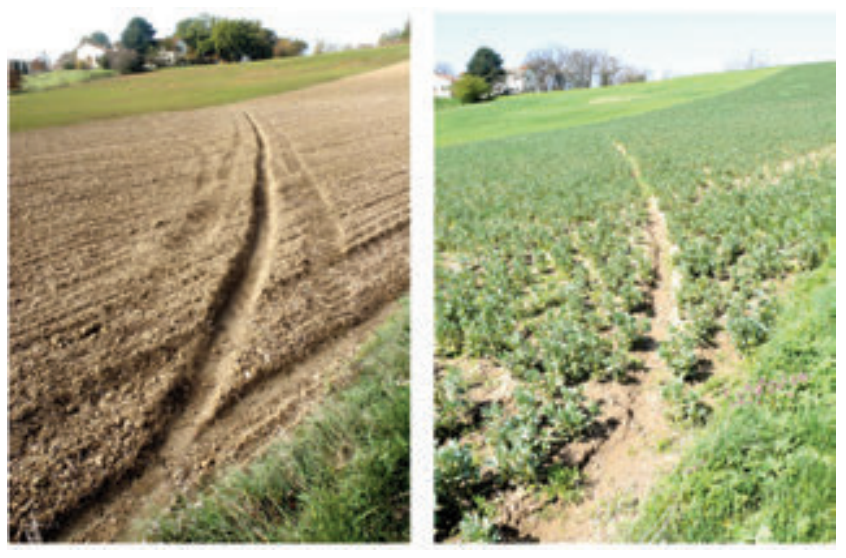

Figura 7. Solco acquaio appena eseguito (a sinistra - ottobre 2009) e alla fine dell'esercizio (a destra - aprile 2010). Si noti la deposizione di sedimento all'interno del solco e la conseguente trattenuta del suolo sul versante.

Tabella 1. Valore medio di deflusso e picco massimo osservati nell'impianto parcellare del CRA-ABP a Vicarello (Volterra) e valori stimati con il metodo razionale.

\begin{tabular}{lcccccccc}
\hline & N. eventi deflusso & & \multicolumn{2}{c}{$\mathrm{m}^{3} \mathrm{~s}^{-1} \mathrm{ha}^{-1}$} & & \\
& & Media & Dev.Std. & Conf. $-95 \%$ & Conf. +95\% & Mediana & Massimo \\
\hline Q stima metodo razionale & 643 & 0,0389 & 0,0454 & 0,0354 & 0,0424 & 0,0205 & 0,2254 \\
Q misura diretta Registraz. elettroniche & 643 & 0,0124 & 0,0191 & 0,0109 & 0,0139 & 0,0027 & 0,0833 \\
\hline
\end{tabular}


Dove $Q=\mathrm{m}^{3}$ sec- $^{1}, \mathrm{~A}=$ area in $\mathrm{m}^{2} ; \mathrm{R}=$ raggio idraulico; $\gamma=1,75$ (Monotti, 1980) e I= 0,025 (pendenza del fondo del solco). Per solchi effettuati con scavafossi 0 con aratro affossatore si ottengono i valori di portata massima di emungimento riportati nella Tabella 3 .

Nella Tabella 4 sono mostrati i valori di picco di deflusso massimo, calcolati con la formula (2), per la superficie scolante compresa fra due solchi acquai contigui, in funzione della lunghezza del solco e della pendenza del versante fino ad un massimo del $25 \%$. Oltre questa pendenza i solchi acquai livellari non solo attuabili a causa dell'instabilità della trattrice.

Confrontando i valori dei picchi di deflusso massimi generati dalle superfici agricole (Tabella 4) con la portata massima dei vari tipo di solchi acquai (Tabella 3) si può calibrare lo standard minimo di affossatura efficace per le situazioni specifiche dell'appezzamento.

Nella progettazione dei solchi acquai si consiglia l'applicazione di un criterio più restrittivo rispetto a quello che considera costante la portata massima di emungimento. Occorre infatti considerare la sezione dei solchi a "fine esercizio"; ovvero la geometria residuale a fine inver- no, quando i solchi non presentano più la profondità iniziale a causa della deposizione di sedimenti e le sponde sono state in parte degradate dagli eventi atmosferici (Figura 7). In un rilievo condotto presso le aziende sperimentali del CRA-ABP (Azienda Santa Elisabetta e Azienda Fagna) nel giugno 2010, si sono rilevati i valori medi delle dimensioni dei solchi acquai (Tabella 5). Occorrerebbe un maggior numero di osservazioni al fine di stabilire la percentuale di degradazione della capacità emungente di diversi tipi di solchi in diversi ambienti del territorio italiano. Comunque, al fine di far fronte a picchi di deflusso che possano verificarsi a fine annata agraria, quando i solchi sono al massimo della loro degradazione, è necessario dimensionare i solchi acquai prendendo in considerazione, a titolo orientativo, una diminuzione di portata media di circa il 30\%, come riportato in Tabella 5. La parziale ostruzione dei solchi acquai non deve essere considerata solo negativamente, infatti l'affossatura primaria svolge anche la funzione di cattura dei sedimenti, con la conseguente riduzione del trasporto a valle del suolo eroso, che così rimane in situ (Figura 7).

Tabella 2. Modello di previsione dl picco massimo di deflusso $\left(\mathrm{m}^{3} \mathrm{ha}^{-1} \mathrm{~s}^{-1}\right)$ in funzione della dimensione e pendenza del bacino.

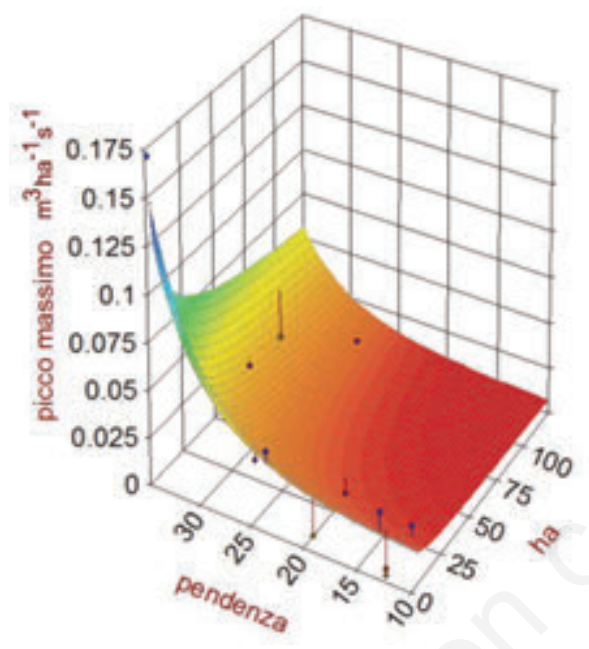

\begin{tabular}{|c|r|r|r|c|c|r|}
\hline Parametro & Valore & Err. St. & t-val. & Conf. $+95 \%$ & Conf. $-95 \%$ & $\mathrm{P}>\mathbf{t}]$ \\
\hline a & $-3,73752632$ & 0,21703284 & 17,227 & 4,22110563 & 3,25394701 & 0,00000 \\
\hline b & $-0,24820406$ & 0,07300852 & 3,400 & 0,41087719 & 0,08553093 & 0,00677 \\
\hline c & $4,79277 \mathrm{e}-05$ & $6,1028 \mathrm{e}-06$ & 7,853 & $3,43296 \mathrm{e}-05$ & $6,15258 \mathrm{e}-05$ & 0,00001 \\
\hline
\end{tabular}

$\mathrm{R}^{2}=0,89 \quad \mathrm{R}^{2}$ Agg. $=0,86 \quad$ Err. St. Stima $=0,01627 \quad \mathrm{~F}=42,20$

\begin{tabular}{|l|c|c|c|c|c|}
\hline Sorgente & Somma Quadr. & G.L. & Quadr.Medio & F Stat. & P>F \\
\hline Regressione & 0,022346 & 2 & 0,01117 & 42,20 & 0,00001 \\
\hline Errore & 0,002647 & 10 & 0,00026 & & \\
\hline Totale & 0,024993 & 12 & & & \\
\hline
\end{tabular}

Tabella 3. Portata massima di diverse tipologie di solchi acquai realizzati con scavafossi o con aratro affossatore a diverse profondità.

\begin{tabular}{lcc} 
Strumento scavafossi & Profondità di lavoravione $(\mathrm{cm})$ & Portata massima del solco $\left(\mathrm{m}^{3} \mathrm{~s}^{-1}\right)$ \\
Rotativo diam., rotore $30 \mathrm{~cm}$ & 15 & 0,0180 \\
Rotativo diam., rotore $32 \mathrm{~cm}$ & 16 & 0,0218 \\
\hline Rotativo diam., rotore $40 \mathrm{~cm}$ & 15 & 0,0238 \\
Rotativo diam., rotore $40 \mathrm{~cm}$ & 20 & 0,0418 \\
\hline Affossatore a sez., trapezia, inclinazione sponde $30^{\circ}$ & 20 & 0,0375 \\
Affossatore a sez., trapezia, inclinazione sponde $30^{\circ}$ & 30 & 0,0841 \\
\hline Affossatore a sez., trapezia, inclinazione sponde $30^{\circ}$ & 40 & 0,1504 \\
Affossatore a sez., trapezia, inclinazione sponde $30^{\circ}$ & 50 & 0,2427 \\
\hline Affossatore a sez., trapezia, inclinazione sponde $30^{\circ}$ & 60 & 0,3626 \\
Aratro affossatore, fosso con inclinazione sponde $45^{\circ}$ & 20 & 0,0117 \\
\hline Aratro affossatore, fosso con inclinazione sponde $45^{\circ}$ & 30 & 0,0385 \\
Aratro affossatore, fosso con inclinazione sponde $45^{\circ}$ & 40 & 0,0895
\end{tabular}




\section{Progettazione dei solchi acquai}

\section{Esercizio svolto}

\section{Quesiti}

Si debba dare assistenza tecnica nella realizzazione dei solchi acquai per l'appezzamento di terreno indicato in Figura 8, indicando all'agricoltore 0 al contoterzista:

la distanza media fra i solchi.

il posizionamento dei solchi.

Il dimensionamento dei solchi.

\section{Svolgimento}

Fase 1: Progettazione della posizione spaziale dei solchi acquai

(Ambienti di lavoro utilizzati: Google Earth, ArcGIS ESRI, Adobe Photoshop, Microsoft Excel)

Si eseguono i seguenti passi:

a) In ambiente Google Earth si ottiene l'immagine dell'appezzamento sul quale realizzare i solchi acquai (Figura 8).

b) Si recupera la carta CTR del sito (di dettaglio, ovvero alla massima scala, secondo la disponibilità regionale) (Figura 9).

c) Si derivano le curve di livello dal file digitale (generalmente in formato AutoCAD) o da digitalizzazione a video e si costruisce il DEM (Modello Digitale delle Quote) con cella di dimensioni pari all'equidistanza delle curve di livello della a CTR dell'area in esame (nel presente esempio: $\mathrm{m} 5 \times 5$ ).

d) $\mathrm{Si}$ determina la pendenza media in gradi sessagesimali (degree) dell'area (con Spatial Analyst Tool>Surface > Slope). Nel caso dell'esempio, la pendenza $(\alpha)$ è risultata pari a $11,75^{\circ}$.

La pendenza media dovrà essere ricavata anche in valore $\%$ in quanto serve nei successivi calcoli. In Excel la sintassi per ottenere la pendenza in \% è la seguente:

$$
=100 *(\operatorname{TAN}(/ 180 * \text { PI.GRECO }()))
$$

Si determina l'equidistanza media delle curve di livello corrispondenti a una distanza inclinata pari a 80 metri (in applicazione dello standard 1.1 a) utilizzato nella seguente formula:

$$
\text { Equidistanza curve }(\mathrm{m})=80 *(\operatorname{Sin}(/ 180 * \pi))
$$

Dove $\alpha=$ pendenza media dell'area in gradi sessagesimali.

Utilizzando un foglio di calcolo Microsoft Excel la formula è la seguente:

$$
=80 *(\operatorname{SEN}(/ 180 * \text { PI.GRECOO }))
$$

Per il caso studio l'equidistanza calcolata è risultata pari a 16,63 m. f) Si tracciano in ArcGIS (con Spatial Analyst Tool >Surface>Contour) le curve corrispondenti alla suddetta equidistanza (Figura 10). Le curce indicheranno in modo approssimativo la posizione dei solchi acquai.

g) Utilizzando il DEM Si applicano in sequenza i due tool Flow Direction e Flow Accumulation (con Spatial Analyst Tool>Hydrology) ottenendo così un'indicazione di come scorre il deflusso sulla superficie del suolo (Figura 11).

h) In ambiente Photoshop si importano le seguenti immagini: l'immagine delle curve di livello (che dovrà risultare in primo piano), l'immagine del flow accumulation (in secondo piano) e l'immagine del terreno di Figura 8 (in terzo piano). Le immagini dovranno risultare perfettamente sovrapposte, ottenendo così tre livelli di Photoshop geograficamente coincidenti.

i) Si agisce sul livello "Flow accumulation", diminuendone l'opacità (aumentandone quindi la trasparenza) in modo da poter intravedere sia le linee di flusso del livello Fow accumulation sia l'immagine del terreno.

j) A questo punto è possibile tracciare "a video" i solchi acquai secondo il seguente criterio: Per ciascuna curva di livello l'inizio del solco deve essere posizionato a quota più elevata della curva medesima $\mathrm{e}$ la parte finale (che confluisce nei fossi collettori e negli alvei naturali, disposti ai bordi dei campi) a quota più bassa (Figura 12) così da consentire il deflusso.

k) Una volta terminata la progettazione dovrà essere fornita l'assistenza in campo in modo da evitare tassativamente che i solchi acquai vengano realizzati con zone di contropendenza nello sviluppo del loro percorso.

Fase 2: Calcolo del picco massimo di deflusso che interesserà i solchi acquai

(Ambienti di lavoro utilizzati: ArcGIS ESRI, Microsoft Excel) Si eseguono i seguenti passi:

a) Si determina in ambiente ArcGIS la lunghezza totale delle curve di livello (sulla Tabella dello shp-file delle curve di livello si richiede sul campo "shape-lenght", utilizzando Statistics, la somma delle lun-

Tabella 4. I valori di picco di deflusso massimo calcolati con la formula (2), per la superficie scolante compresa fra due solchi acquai contigui, in funzione della lunghezza del solco, della pendenza del versante e della spaziatura fra solchi.

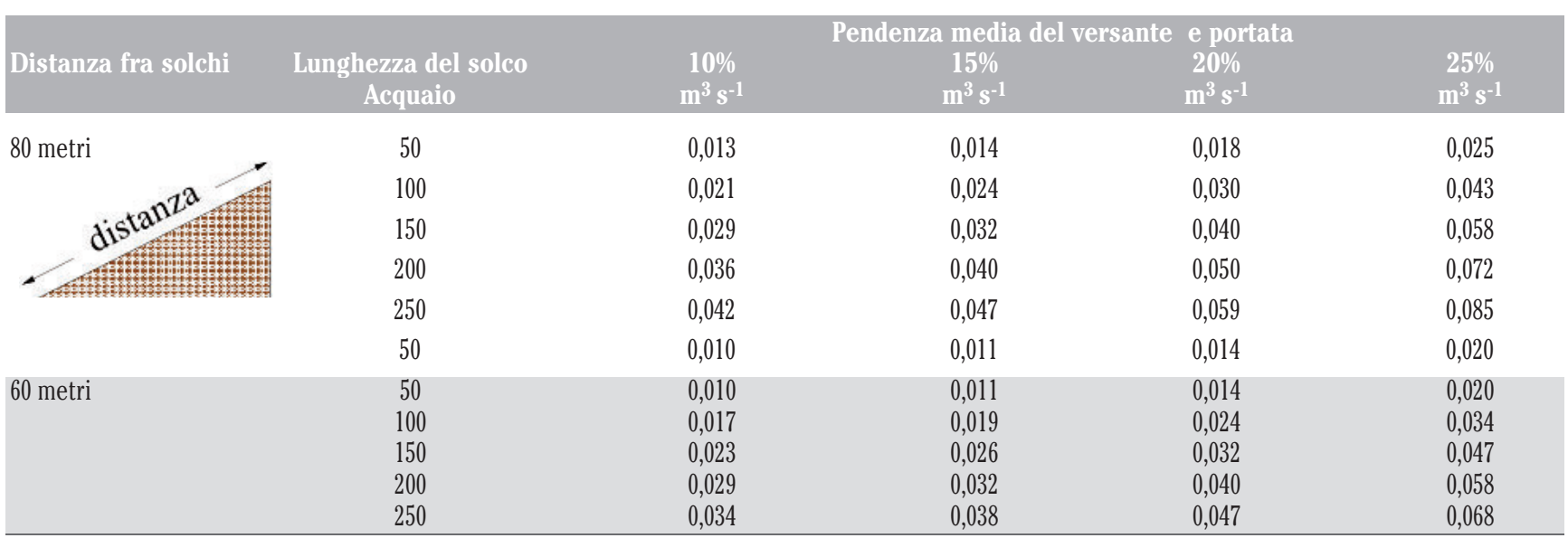


ghezze. Nel presente esempio la somma è risultata pari a 2416,19 metri.

b) Si calcola l'area fra solchi contigui (valore medio).

Si calcola l'area fra solchi contigui (valore medio).

area ha $=($ (lunghezza solchi $\mathrm{m} /$ numero di curve di livello)

* distanza solchi m)/10000)

Nel nostro esempio la formula in Excel sarà:

ha $=(2416.19 \mathrm{~m} / 6$ curve $* 80 \mathrm{~m}) / 10000$ con risultato ha=3,22 (eq. 9)

c) Si può quindi procedere al calcolo del picco massimo di deflusso applicando la formula (2). Utilizzando il foglio di calcolo Microsoft Excel, la sintassi della formula è la seguente:

$$
\begin{aligned}
= & \operatorname{EXP}((-3.73752632159-0.24820405748 * \mathrm{LN} \\
& (\text { area ha })+0.00004792768 *(\text { Pend } \%) 3))
\end{aligned}
$$

Che, applicando i valori ricavati per il presente esempio, diviene

$$
\begin{aligned}
& =\mathrm{EXP}((-3.73752632159-0.24820405748 \\
& \left.\left.{ }^{*} \mathrm{LN}(3.22)+0.00004792768^{*}(21.25)^{\wedge} 3\right)\right)
\end{aligned}
$$

Con il seguente risultato risultato: portata massima di emungimento da parte del singolo solco $Q=0,0282 \mathrm{~m}^{3} \mathrm{~s}^{-1}$.

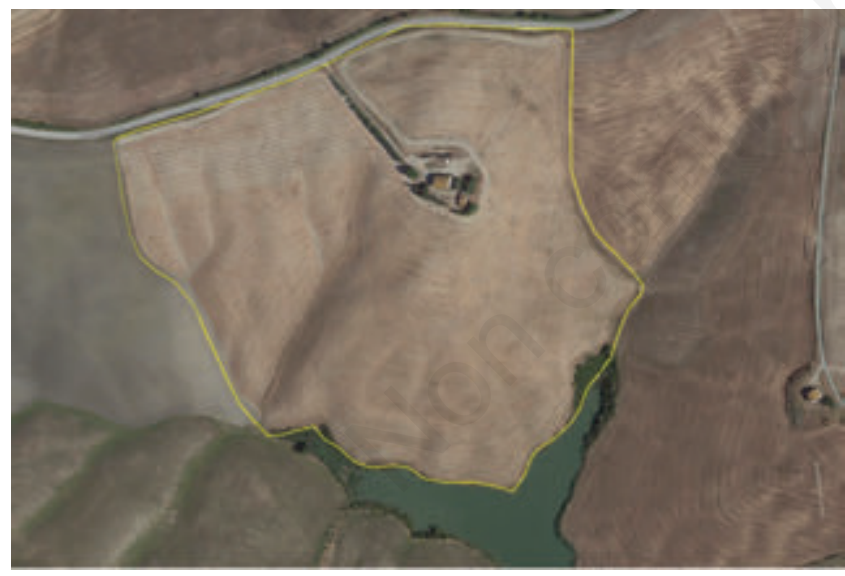

Figura 8. Area di progettazione dei solchi acquai.
Fase 3: Calcolo della dimensione ottimale dei solchi ed individuazione dello strumento affossatore da utilizzare

d) Il valore ottenuto $0,0282 \mathrm{~m}^{3} \mathrm{~s}^{-1}$ deve essere aumentato del $30 \%$ per tener conto della perdita di capacità regimante, per cui si ottiene il valore di $0,0367 \mathrm{~m}^{3} \mathrm{~s}^{-1}$. Quest'ultimo valore viene utilizzato in Tabella 3 per ricavare la profondità dei solchi e lo strumento da utilizzare.

In questo esempio il valore in Tabella che più si avvicina (per eccesso) al valore ricavato $0,0367 \mathrm{~m}^{3} \mathrm{~s}^{-1}$ è $0,0418 \mathrm{~m}^{3} \mathrm{~s}^{-1}$. In corrispondenza di tale valore la Tabella indica che si dovrà utilizzare uno scavafossi con diametro del rotore di $40 \mathrm{~cm}$ e profondità di esecuzione dei solchi di almeno $20 \mathrm{~cm}$.

\section{Azioni agronomiche per la protezione del suolo nella riduzione dell'erosione: lo Standard $\mathbf{1 . 2}$ (impegno h sub v) adottato in luogo dello Standard 1.2 (impegno h sub iv)}

La condizionalità aggiunge ulteriori impegni agronomici ad integrazione dell'obbligo di realizzazione dei solchi acquai temporanei in terreni declivi che manifestano fenomeni erosivi evidenziabili dalla presenza di incisioni diffuse (rigagnoli) in assenza di sistemazioni. Fra

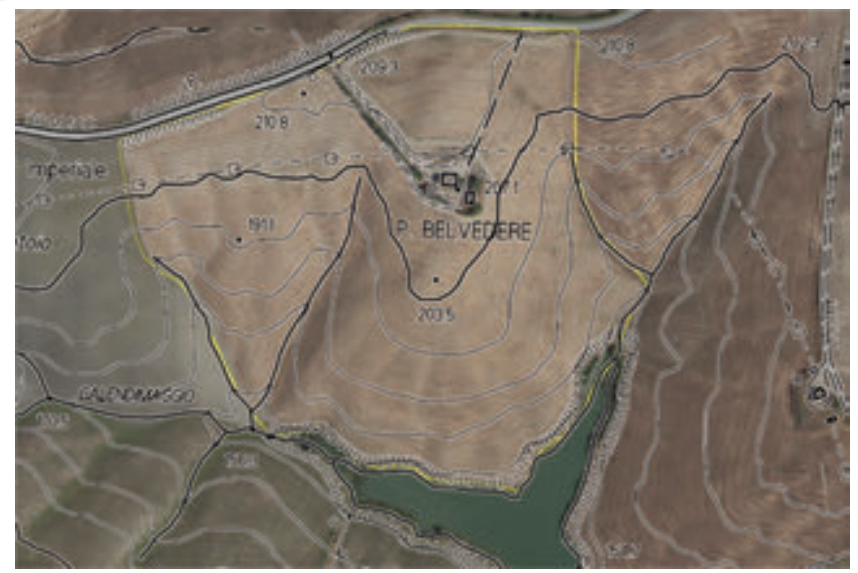

Figura 9. Curve di livello dalla CTR2000 Regionale sovraimposte all'immagine Google Earth.

Tabella 5. Degradazione dei solchi acquai presso le aziende sperimentali Santa Elisabetta e Fagna del CREA-ABP.

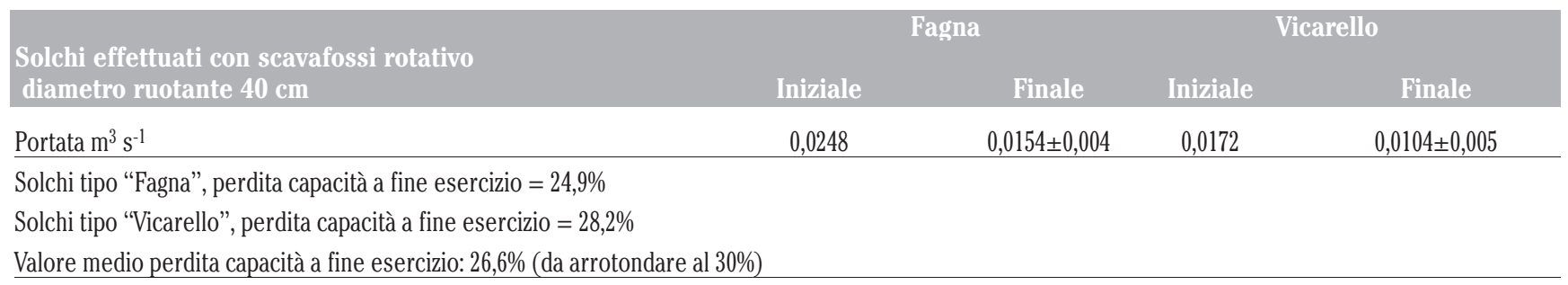


questi, l'impegno previsto dallo standard 1.2 (impegno hub iv.) di assicurare la copertura vegetale del suolo per almeno 90 giorni consecutivi, nell'intervallo di tempo compreso tra il 15 settembre e il 15 maggio successivo, 0 in alternativa l'impegno previsto dallo standard 1.2 (impegno h sub v.) di adottare tecniche per la protezione del suolo (come ad esempio la discissura in luogo dell'ordinaria aratura, lasciare i residui colturali, ecc.). È molto importante sottolineare la sinergia fra le lavorazioni del suolo ed i solchi acquai nel governo delle acque e nella riduzione dell'erosione. Nelle terre declivi a tessitura da franco-limosa-argillosa a argillosa anche le lavorazioni del terreno possono contribuire efficacemente alla protezione del suolo dall'erosione, se eseguite secondo precisi schemi che dipendono dal clima e dalle caratteristiche di stazione. In modo particolare, su questi suoli, è necessario eseguire la lavorazione in tempera asciutta, in quanto essa favorisce la formazione di macro e microaggregati di buona stabilità che consentono l'infiltrazione e la conseguente diminuzione dello scorrimento delle acque (Landi, 1984).

Evidentemente, come anche Landi (1984) sottolineava, occorre avere una visione complessiva delle problematiche che riguardano le diverse aree agricole infatti non si può generalizzare che le lavorazioni diminuiscano lo scorrimento superficiale e l'erosione. In particolare, riguardo allo standard $1.2_{\text {(impegno h sub v.) }}$, occorre considerare l'influenza delle lavorazioni sulla stabilità dei versanti e sul pericolo di frane superficiali, che possono essere indotte dalla troppa pendenza e dall'eccesso idrico all'interfaccia fra l'orizzonte arato e la suola di aratura (zona compattata dagli strumenti di lavorazione del suolo).

\section{Conoscenze e indicazioni}

Limitando l'indagine all'effetto dell'aratura sui deflussi e sull'erosione, si riportano i risultati di una ricerca condotta fra il 1982 e il 1984 da Basso et al. (1986) in una zona collinare della Basilicata (700 m s.l.m.), su suolo Franco Limoso-argilloso con pendenza del 14\%, su parcelle di $600 \mathrm{~m}^{2}$, con tre ripetizioni e con distribuzione a blocchi.

La ricerca ha accertato gli effetti di quattro metodi di lavorazione del terreno (aratura profonda a $40 \mathrm{~cm}$, aratura superficiale a $20 \mathrm{~cm}$, fresatura e non lavorazione) sull'erosione del suolo ed ha messo in evidenza il migliore effetto regimante dell'aratura alle due profondità, rispetto alla fresatura ed alla non lavorazione. L' erosione è stata nell'ordine, di 3,4 - 3,5 $\mathrm{t} \mathrm{ha}^{-1}$ rispettivamente per l'aratura a $40 \mathrm{~cm}$ e a 20 ; mentre per la fresatura e la non lavorazione è risultata pari rispettivamente a 3,7 e $4,1 \mathrm{t} \mathrm{ha}^{-1}$, Anche i deflussi sono risultati più bassi nelle tesi arate rispetto a quelle fresate e a non lavorazione, Infatti sono risultati mediamente $122 \mathrm{~mm}$ e $146 \mathrm{~mm}$ per l'aratura a $40 \mathrm{~cm}$ e a $20 \mathrm{~cm}$ e 162,6 $\mathrm{mm}$ e 188,0 mm, per la fresatura e la non lavorazione. Nelle ricerche condotte dal CREA-ABP in una prova parcellare nell'azienda Santa Elisabetta del CREA (Volterra), nel periodo 1997-1999, su grano, sono stati messi a confronto gli effetti sull'erosione e sui deflussi di 4 tipi di lavorazione del suolo: aratura a $45 \mathrm{~cm}$, a $30 \mathrm{~cm}$, a $15 \mathrm{~cm}$, discatura (erpice Morgan), Nelle tesi arate il suolo è stato successivamente fresato per preparare il letto di semina. I risultati ottenuti mostrano (Figura 13) che nell'evento estremo di $12 \mathrm{~mm}$ caduti in $3^{\text {h }} 53^{\text {' la disca- }}$ tura è stata più efficace dell'aratura con aratro a versoio nel proteggere il suolo dall'erosione. Una spiegazione a questi risultati può essere fornita considerando che la discatura non ha rinettato bene il terreno dalle malerbe e ha garantito un certo grado di copertura del suolo; inoltre il suolo discato presenta una zollosità (rugosità) superiore a quella del suolo arato e preparato per la semina. Poiché è dimostrato che l'erosione del suolo viene efficacemente contrastata dal mantenimento della rugosità del suolo (zollosità) e della copertura con i residui della coltura precedente, occorre evitare (e forse abolire) l'eccessivo amminutamento del suolo, in modo particolare occorre evitare la fresatura e la rullatura del terreno post semina nelle zone declivi a pendenza $>5 \%$.

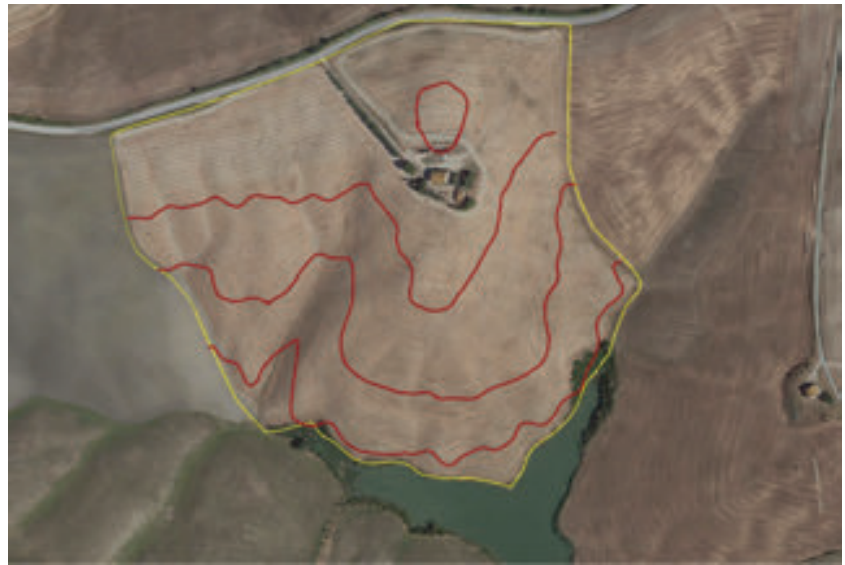

Figura 10. Curve di livello con equidistanza $80 \mathrm{~m}$.

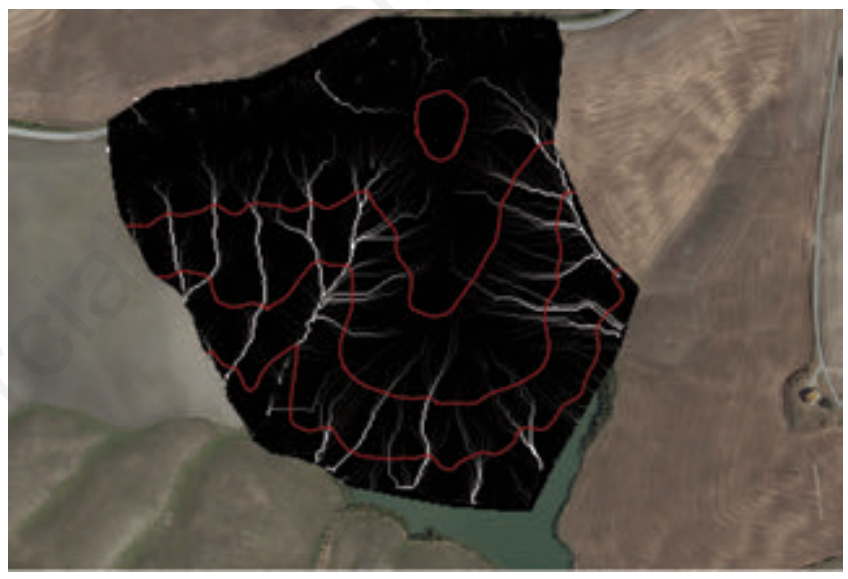

Figura 11. Curve di livello con equidistanza $80 \mathrm{~m}$ sovraimposte alle linee di flusso.

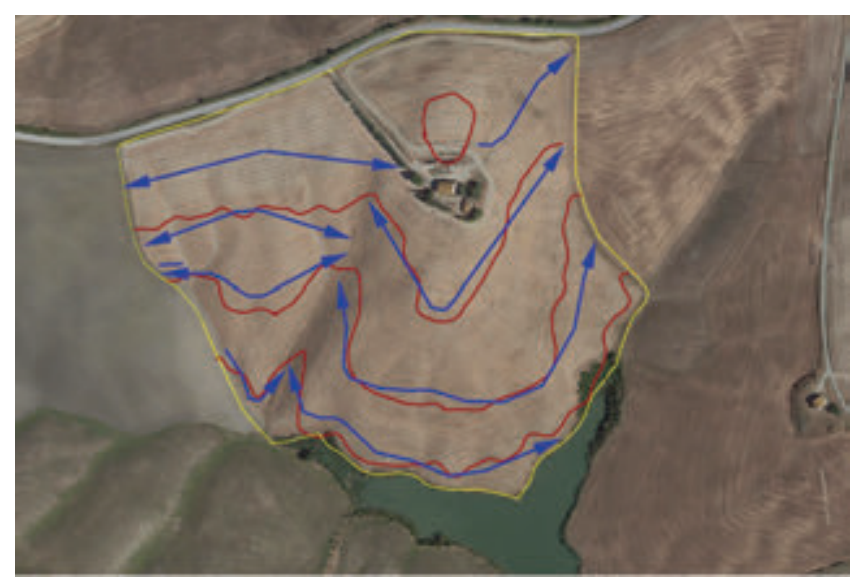

Figura 12. Progetto dei solchi acquai. Il verso delle frecce indica la direzione del flusso idrico incanalato dai solchi acquai e quindi il verso dell'inclinazione dei solchi. 

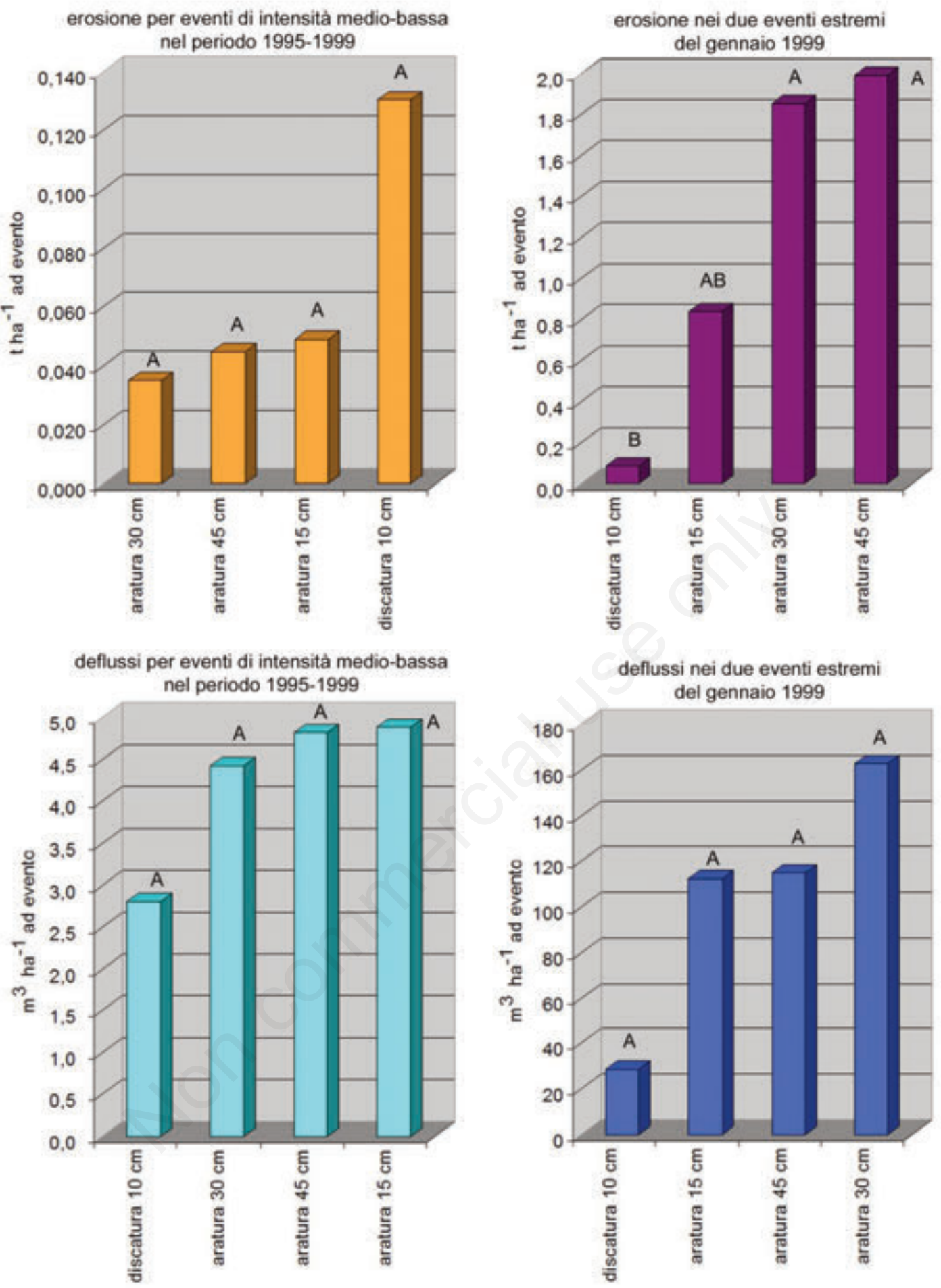

Figura 13. Deflussi ed erosione osservati a Vicarello (Volterra) su tesi coltivate a grano con diversa lavorazione del suolo. Valori delle lettere differenti indicano una differenza fra le medie significativa, $P \leq 0,005$.

\section{Bibliografia}

Bazzoffi P, Francaviglia R, Neri U, Napoli R, Marchetti A, Falcucci M, Pennelli B, Simonetti G, Barchetti A, Migliore M, Fedrizzi M, Guerrieri M, Pagano M, Puri D, Sperandio G, Ventrella D, 2015. Environmental effectiveness of GAEC cross-compliance Standard 1.1a (temporary ditches) and $1.2 \mathrm{~g}$ (permanent grass cover of set- aside) in reducing soil erosion and economic evaluation of the competitiveness gap for farmers. Ital. J. Agron. 10(s1):710.

Bazzoffi P, Pellegrini S, 1992. Caratteristiche delle piogge influenti sui processi erosivi nel periodo 1964-1990 in un ambiente della valle dell'Era (Toscana), evoluzione climatica e modelli previsionali. Ann. Ist. Sper. Studio Difesa Suolo 20:161-182.

Bazzoffi P, Pellegrini S, Chisci G, Papini R, Scagnozzi A, 1997. Erosione e deflussi a scala parcellare e di bacino in suoli argillosi a diversa 
utilizzazione nella val d'Era. Agricoltura Ricerca 170:5-20.

Buffoni L, Brunetti M, Mangianti F, Maugeri M, Monti F, Nanni T, 2003. Ricostruzione del clima italiano negli ultimi 130 anni e scenari per il XXI secolo. Atti Workshop CLIMAGRI - Cambiamenti climatici e agricoltura, Cagliari.

Chisci G, Boschi V, 1988. Runoff and erosion control with hill farming in the sub-coastal Apennines climate. Soil Till. Res. 12: 05-120.

Gasparini M, 1959. Aspetti agronomici della meccanizzazione collinare in Toscana. Atti Conv. Meccanizzazione collinare, Firenze, pp 39-58.

Gasparini M, 1970. Evoluzione delle sistemazioni idraulico-agrarie nelle terre declivi. In: Difesa e conservazione del suolo dalle erosioni idrogeologiche. Istituto di Tecnica e Propaganda Agraria, Roma.

Landi R, 1978. Struttura e miglioramento dei terreni argillosi. pp 49-80 In: L'utilizzazione dei terreni argillosi dell'Appennino. ISEA, Bologna.

Landi R,1984. Regimazione idraulico-agraria e conservazione del suolo. Riv. Agron. 18:147-174.
Landi R, Mattei Scarpaccini F, Raso E. 1982. Interventi sistematori nei terreni collinari. Atti Convegno Finale C.N.R. Conservazione del Suolo, Roma.

Lisa L, Parena S, 1994. Erosione in vigneti collinari e tecniche per ridurre il fenomeno. Attività svolta nel 1992-1993 nell'ambito della convenzione con la Regione Piemonte. Disponibile a: http://web.tiscalinet.it/accagri/Pages/poster2e.htm

MiPAAF, 2009. Testo consolidato del decreto ministeriale n.30125 del 22 dicembre 2009. Supplemento ordinario alla Gazzetta Ufficiale n. 303 del 31 dicembre 2009 - Serie generale.

Monotti M, 1980. Affossatura, dimensionamento, realizzazione. Italia Agricola 117: 72-84.

Scoccimarro E, Gualdi S, Bellucci A, Zampieri M, Navarra A, 2013. Heavy precipitation events in a warmer climate: results from CMIP5 Models. J. Climate 26:7902-7911. 
